\title{
PERCUTANEOUS EDGE TO EDGE MITRACLIP THERAPY IN THE MANAGEMENT OF MITRAL REGURGITATION
}

\author{
Trayan Tsvetkov, Svetoslav Georgiev \\ Department of Cardiology, Medical University of Varna, Bulgaria
}

\begin{abstract}
Mitral valve regurgitation (MR) is an important clinical issue as MR represents $>30 \%$ of native valve diseases. Patients with symptomatic MR have a poor prognosis, with a $5 \%$ annual mortality rate in the absence of surgery. Optimal medical management can improve symptoms of heart failure but does not affect survival. Therefore, surgery is recommended by the current guidelines for patients with symptomatic severe MR or asymptomatic severe MR with evidence of left ventricular (LV) dysfunction or dilation. Despite the guidelines, a recent European survey established that one-half of the patients are not referred for surgery, mainly because of advanced age and the presence of comorbidity. Mitral valve (MV) repair is the preferred surgical strategy whenever feasible and is associated with lower morbidity and mortality and better preservation of LV function, compared with MV replacement. Reported in-hospital mortality rates range from $1 \%$ to $2 \%$ in low-risk patients, increasing up to $25 \%$ in high-risk or elderly patients. Therefore, new percutaneous techniques are developed to avoid surgery in high-risk patients (1).
\end{abstract}

Keywords: mitral, regurgitation, Mitraclip

The transcatheter edge-to-edge MV repair using the MitraClip system creates a double MV orifice by means of a clip in the mid portion of the 2 leaflets and mimics the surgical procedure introduced by $\mathrm{Al}-$ fieri et al. (1). Edge-to-edge surgical repair of the mitral valve with annuloplasty (or without annuloplasty in selected patients) has been shown to afford favourable outcomes in patients with degenerative or functional MR (FMR) (1).

Address for correspondence:

Trayan Tsvetkov

Department of Cardiology

Medical University of Varna

55 Marin Drinov Str.

9002 Varna, Bulgaria

e-mail:trayan_tsvetkov@hotmail.com

Received: November 16, 2014

Accepted: March 11, 2015
Functional mitral regurgitation is a direct consequence of underlying left ventricular dysfunction and annular dilation which secondarily impairs the coaptation of otherwise structurally normal leaflets. Surgical correction of FMR has been shown to improve functional class and left ventricular remodelling, but a survival benefit has yet to be shown (1). Additionally, even with current surgical annuloplasty techniques, up to $35 \%$ of treated patients have moderate or greater MR reoccur within 1 year of surgery (1).

The anatomical criteria are mainly obtained by transesophageal echocardiography. All these criteria are preliminary and the exclusion criteria could represent a relative contraindication for the procedure (2). The main criterion is jet location. The currently designed MitraClip ${ }^{\text {tix }}$ can only be used to treat MR originating from the central portion of the valve (A2- 
P2 segments). Baseline valve area is also an important factor associated to post-procedural trans-mitral gradient. MitraClip ${ }^{\text {tw }}$ should not be implanted in case of a valve area below $4 \mathrm{~cm}^{2}$. For the same reason, the procedure is not indicated in case of limited leaflet pliability (as in rheumatic disease). Also, any leaflet organic disease (perforations, calcifications, erosions, etc.) should be considered a relative contraindication to the procedure.

As for other transcatheter valve procedures, MitraClip $^{\text {tw }}$ interventions are ideally performed by a multidisciplinary team composed of surgeons, interventional and non-interventional cardiologists and anesthesiologists.

The surgical technique of Alfieri involves the use of suture to convert a regurgitant mitral orifice into a competent double orifice valve with decreased or eliminated regurgitation. The MitraClip device, which was designed to mimic an edge-to-edge repair, consists of a percutaneously delivered MRI-compatible cobalt chromium implant with two arms and two grippers which are used to grasp the opposing free edges of the anterior and posterior leaflet in order to improve leaflet coaptation and generate a double-orifice valve. Each arm is $4 \mathrm{~mm}$ wide and $8 \mathrm{~mm}$ long, and the device is covered in a polyester fabric to promote tissue in-growth. Complete encapsulation of the device occurs by 12 weeks $(2,3)$.

The procedure is performed in the catheterization laboratory under general anaesthesia for patient comfort (due to use of TEE during the procedure), but more importantly to eliminate patient movement and to perform controlled breath-holding during the MitraClip grasping step, thereby allowing accurate placement and controlled leaflet insertion.

MitraClip therapy is highly compatible with other minimally invasive procedures that are often applied to patients with MR. For example, access to the left atrium is not restricted for atrial fibrillation ablation procedures, and access to the coronary sinus is preserved for biventricular lead ICD placement or for future coronary sinus mitral annuloplasty devices. Biventricular pacing and cardiac resynchronization therapy is an important treatment for patients with ischaemic cardiomyopathy and can reduce FMR acutely and chronically in selected patients. Greater than one-half of responders to CRT have sus- tained reductions in MR by at least one grade for 6 months or longer (1).

The EVEREST I, EVEREST II and REALISM clinical studies $(2,3)$ have evaluated the MitraClip device in patients diagnosed with mitral regurgitation. The clinical data from these trials have demonstrated that the MitraClip device and the procedure is safe. The MitraClip device has also been shown to effectively reduce MR, improve left ventricular function, and improve clinical outcomes, as evidenced by improvements in NYHA Functional Class and improvements in Quality of Life (QoL) in patients with significant MR.

The EVEREST I Feasibility Study evaluated the safety of the MitraClip device $(2,3)$. The EVEREST II Randomized Clinical Trial compared the safety and effectiveness of the MitraClip device to conventional open-heart surgical mitral valve repair (3). The EVEREST II High Risk Registry evaluated the safety and effectiveness of the MitraClip device in high risk surgical candidates (3). The EVEREST II REALISM Continued Access Registry evaluate the safety and effectiveness of the MitraClip device in high risk and non-high risk patients (3). Follow-up on patients enrolled in the EVEREST I and EVEREST II clinical trials, including REALISM, continued for 5 years.

The purpose of the Cardiovascular Outcomes Assessment of the MitraClip Percutaneous Therapy for Heart Failure Patients with Functional Mitral Regurgitation(COAPT) Trial is to confirm the safety and effectiveness of the MitraClip System for the treatment of moderate-to-severe or severe functional mitral regurgitation (FMR) in Symptomatic Heart Failure Subjects (5).

Data from ACCESS-EU $(5,6)$ demonstrated that in real-world, post-approval experience in Europe, patients undergoing the MitraClip therapy have a predominantly high surgical risk, elderly patients who are mainly affected by functional MR, a type of MR in which a damaged heart impairs the performance of a normal mitral valve. ACCESS-EU showed that in this patient population, the MitraClip procedure demonstrated low rates of hospital mortality and adverse events and provided significant improvements in day-to-day quality of life at one year following treatment. 
The first clinical trials with the MitraClip showed very promising results regarding the feasibility and safety of the device, and functional improvement of the patient $(2,3)$.

As a result of MR reduction, diminished plasma NT-proBNP levels, accompanied with significant improvements in QoL and functional capacity, which may be the primary goal in this challenging high-risk population. The improvement in objective parameters shows that the observed clinical effect can be attributed to improvements beyond a placebo effect. NYHA functional class improved in $89 \%$ of this population, which is in line with previous reports. The EVEREST II trial showed similar improvements in NYHA functional class and QoL in both the clip and surgery subgroups at 12 months (3).

Percutaneous MV repair seems to be associated with positive reverse LV remodeling a significant increase in LVEF, decrease in LVEDD, and obvious improvements in LV volumes (5). The question remains whether this will affect long-term prognosis, as previous reports showed no evidence that surgery for functional MR prolongslife, LV reverse remodeling.

\section{CONCLUSION}

Percutaneous approaches become a valuable treatment option for an important number of patients with severe (especially functional) MR who cannot undergo surgery. In the future, the field of percutaneous transcatheter MV repair will undoubtedly evolve exponentially, because functional MR is primarily a disease of the $\mathrm{LV}$, a combination of the edge-to-edge repair with other percutaneous techniques addressing the annulus and the LV itself will be necessary to provide durable correction and satisfactory long-term outcomes.

\section{REFERENCES}

1. Carabello BA.The current therapy for mitral regurgitation. J Am Coll Cardiol. 2008;52:319-326.

2. Feldman T, HS Wasserman, HC Herrmann, et al. Percutaneous mitral valve repair using the edgeto-edge technique: six-month results of the EVEREST phase I clinical trial. J Am Coll Cardiol. 2005;46:2134-2140.

3. Feldman T, S Kar, M Rinaldi, et al., EVEREST Investigators. Percutaneous mitral repair with the MitraClip system: safety and mid-term durability in the initial EVEREST (Endovascular Valve Edgeto-Edge Repair Study) cohort. J Am Coll Cardiol. 2009;54:686-694.

4. Herrmann HC, S Kar, R Siegel, et al. EVEREST Investigators. Effect of percutaneous mitral repair with the MitraClip device on mitral valve area and gradient. EuroIntervention. 2009;4:437-442.

5. Tamburino C, GP Ussia, F Maisano et al. Percutaneous mitral valve repair with the MitraClip system: acute results from a real world setting. Eur Heart J. 2010;31:1382-1389.

6. Van den Branden BJ, MC Post, MJ Swaans, et al. Percutaneous mitral valve repair using the edgeto-edge technique in a high-risk population. Neth Heart J. 2010;18:437-443. 